\title{
Discussion to the papers by G. Walters, M. D. A. Vickers, and J. F. Riordan, G. Walters and W. D. S. McLay
}

KeANEY. The last figures emphasize the importance of correlating clinical observations and apparently objective signs, and the need to treat patients rather than isolated readings such as the CVP.

If a number of us here were asked to write the characteristics of a good pulse volume, what would we say? Is it a central pulse, a peripheral pulse or what? Is it in a thin arm or a fat arm? If you have a patient with a rapid respiratory rate and a good pulse volume have you measured the $\mathrm{PCO}_{2}$ ? In the case of the woman dying of ulcerative colitis where there was a continually rising respiratory rate, apparent hypoxia and a good pulse volume, there was no indication of the $\mathrm{PCO}_{2}$.

WALTERS. It is very difficult to describe what is meant by a 'good pulse volume'. It is determined by feeling the radial pulse. It is something that can be taught at the bedside as a number of people in this audience could testify. Certainly there may be an easily palpable femoral pulse when the radial pulses are impalpable. Changes in pulse volume in response to transfusion are much easier to appreciate. Dr Riordan and I do not always agree as to what is a good pulse volume, but we always agree on changes occurring in response to transfusion. If there is doubt and the CVP is within the normal range, I would transfuse.

With regard to $\mathrm{PCO}_{2}$, when the blood pressure was falling and the cardiac output high, blood $\mathrm{pH}$ was $7 \cdot 42$ and $\mathrm{PCO}_{2} 33 \mathrm{mmHg}$. The cardiac output was high $(7.5$ $1 / \mathrm{min}$ ) and the pulse volume was reflecting this. Patients with rapid respirations often have a respiratory alkalosis. Such changes contribute little to the diagnosis or management unless there is a really severe acidosis, which should be corrected.

VICKERS. Changes in peripheral circulation during anaesthesia are clearly indicated by Johnstone's digital plethysmograph which measures finger pulsation.

Robinson. An ECG on the same trace is also useful to give some indication of circulation time.

STEWART. As medical students we were taught to take the history first and to make the physical examination second, and then to do special investigations. Although we are now focusing attention on special investigations, it is very important to go back to the history and to find out what operation a patient has had. If sepsis is suspected it should be treated empirically with an antibiotic 'cocktail'. The simple matter of taking a patient's history is of absolutely first and vital importance. Another useful empirical measure, if the heart is not functioning well, is intravenous digitalization.

WALTERS. When a patient suddenly collapses and does not have a high CVP, peritonitis should be assumed if he has had an abdominal operation. The belly can be soft, with no rigidity and no abdominal pain, and it is not until $24 \mathrm{hr}$ later that everyone agrees it is peritonitis. With a massive pulmonary embolus you would not expect to hear a pleural rub because infarction takes time to develop. A rub is more likely to be due to inflammation under the diaphragm or from an underlying pneumonia.

PEARSON. Digitalization in patients with persistently high CVP but otherwise normal hearts can be valuable. A recent patient with an $E$. coli septicaemia who was transfused up to a high normal CVP showed no improvement until he was digitalized when the CVP fell dramatically, the peripheral vessels dilated and he secreted urine.

SCHRÖDER. Phenoxybenzamine may be dangerous in cardiogenic shock especially if the patient is already hypotensive.

We did not find any significant changes in cardiac output, CVP or pulmonary artery pressure in twenty patients with recent myocardial infarction who were given digoxin intravenously. There was, however, some inotropic effect, as shown by improved contractility, judged by the pre-ejection period of left ventricular systole.

RIORDAN. Our experience in cardiogenic shock due to myocardial infarction is that digitalis has little effect. We have not used it in young patients with normal hearts. The sort of response described has been seen mostly in elderly patients, who probably have some degree of myocardial abnormality.

The place of phenoxybenzamine in myocardial infarction is uncertain, and it has only been effective in two of seven patients. Both had a metabolic acidosis and hypotension lasting 6-7 hr, not reversed by digitalis. In the patients who showed no response, the drug was given rather rapidly in 1-2 hr and this sometimes caused alarming wheezing. Three patients died rapidly and at autopsy were found to have severe pulmonary oedema. It is wise to give the drug slowly, but how to decide which patients will benefit remains a problem.

Pentecost. Palpation of radial arteries can be a source of error in shocked patients. As people get older their arteries change in character, and a good pulse volume can be found with relatively poor peripheral perfusion.

Measurements of cardiac output by ear-piece oximeters can also be misleading in the presence of shock. It is possible to change the vascular bed of the ear by infusing noradrenaline and other vaso-constrictors, and it is probably unreliable as a measure of cardiac output in shock. Some patients who develop pulmonary oedema do improve on digitalis alone, and this is one of the things to come out of the recent studies by McNicol (Kirby, McNicol \& Tattersfield, 1968) and by Nixon (Nixon, Taylor \& Morton, 1968) at Charing Cross Hospital.

There are obviously two completely distinct types of shock following myocardial infarction: one with elevated and the other with normal or lowered left ventricular filling pressure. This may be the key to the whole value of transfusion and peripheral vasodilators in the management of shock after myocardial infarction. Patients can 
be killed with phenoxybenzamine as with any other drug, if you do not know what you are doing. If phenoxybenzamine is given it is necessary to stay with the patient and be prepared to expand the circulation as the arterial pressure falls.

VICKERS. Do you mean that it is unwise to rely on the radial pulse?

Pentecost. The trouble is that we tend to be obsessed with haemodynamic measurements. Perhaps we should forget about arterial and venous pressures and cardiac output and try to find some other criterion for saying that the treatment is doing some good. It is no good measuring the cardiac output if we don't know where it is going. The easiest thing to achieve in almost any hospital nowadays within a matter of 2 or $3 \mathrm{~min}$ is an arterial assessment of $\mathrm{pH}$ and $\mathrm{PCO}_{2}$. No haemodynamic data are of sufficient value in treating myocardial infarction.

RoBINSON. Speaking as an 'agricultural anaesthetist' might we agree to regard the heart as a constant volume pump rather than a constant pressure pump, so that the pulse volume becomes a little more important?

Walters. The limitations of $\mathrm{pH}$ and $\mathrm{PCC}_{2}$, etc., as indications of severity must be stressed. Some patients with bacterial infections may behave like cases of pulmonary embolism and collapse dramatically. They may not have a high $\mathrm{PCO}_{2}$ initially and it is some time before they develop a metabolic acidosis; the absence of such changes must not mislead one into a leisurely approach to treatment.

Dr Pentecost has already stressed that people must know what they are doing. Patients have had trans- fusions stopped because the CVP was apparently high because of venous thrombosis, the external jugular vein being distended on one side only. Totally erroneous readings of CVP have been made because a catheter has become blocked. I have also seen patients with a supposedly poor pulse volume because both wrists have not been felt.

Hopkin. It may be difficult to feel the pulse when a patient is shocked because of the high vasomotor tone. Even though the pulse is difficult to feel, the systolic blood pressure may be 100 or more. If a vasodilator drug is given, the peripheral circulation improves and the pulse becomes more easily palpable, although the systolic pressure may fall to 80 . Many years ago, before the days of routine intravenous infusion, I had to anaesthetize patients bled out from a ruptured ectopic who were usually pulseless; but, as soon as the surgeon removed the blood clot from the abdomen, the pulse always became palpable. Presumably irritation of autonomic nerve endings in the peritoneal cavity by blood clot reflexly induced high vasomotor tone so that the pulse could not be felt.

\section{References}

Kirby, B.J., McNicol, M.W. \& TAtTersfield, A.E. (1968) Left ventricular pressures in two patients with myocardial infarction. Lancet, i, 944.

Nixon, P.G.F., TAYlor, D.E. \& Morton, S.D. (1968) Left ventricular pressure in cardiogenic shock treated by dextrose infusion and adrenaline. Lancet, i, 1230. 